\title{
Considering social ergonomics: the effects of HIT on interpersonal relationships between patients and clinicians
}

\author{
Enid Montague, $\mathrm{PhD}$ ' Onur Asan, MS \\ ${ }^{1,2}$ UW-Madison, Industrial and System Engineering, 3017 Mechanical Engineering Building 1513 University \\ Avenue Madison, WI 53706-1572, USA
}

\begin{abstract}
This study explored physicians' interactions with EHRs to understand the qualities that contribute to patient satisfaction with their use of the technologies and patient satisfaction with physician. Video-taped observations of 100 medical consultations were used to distinguish interaction patterns between physicians and EHRs. Quantified observational methods were used to contribute to ecological validity. Ten primary care physicians and 100 patients from five clinics participated in the study. Visits were videotaped and coded using an objective coding methodology to understand how physicians interacted with electronic health records. Results indicate, a variety of EHR interaction styles may be effective in providing patient-centered care.
\end{abstract}

\section{Introduction}

Widespread implementation an usage of electronic health records (EHRs) and other health information technologies may improve the quality and efficiency of health care in United States. ${ }^{1,2}$ The American Recovery and Reinvestment Act (ARRA) provided a $\$ 19$ billion fund to promote the adoption of EHRs with the requirement of "meaningful use". This requirement states that EHRs should be used effectively and provide quality and efficiency in the health care system. ${ }^{3}$ Despite this goal, a National Research Council (NRC) report indicates that current EHR technologies are poorly designed; and more specifically the technology does not compliment care providers cognitive capabilities and needs. ${ }^{4}$ Furthermore, the NRC report states that current EHRs are not designed with human-computer interaction and human factors and ergonomics design principles, which contributes to inefficient use. ${ }^{4}$

Physician-patient communication is a key element in health care delivery. It is also a significant contributor to patient outcomes such as, patient satisfaction, adherence, rapport and trust. ${ }^{6,7}$ A study on physician-patient interaction found that physicians who maintained high levels of eye contact had higher patient outcomes such as satisfaction and perception of physician empathy. ${ }^{8}$ In addition, computer use could potentially improve physician-patient communication $^{9}$ and increase patient satisfaction. ${ }^{10}$ On the other hand, computer use may reduce positive communication cues such as, eye contact and could make patients feel disengaged or feel that their physicians are less attentive. ${ }^{11}$ Computer use may also increase physicians' mental workload, making it difficult for them to simultaneously enter data and engage in patient-centered care. ${ }^{12}$ Despite its importance, the effects of EHR use on physicianpatient interaction and communication are not covered in the core requirements of meaningful use. The NRC report demonstrates a need for new guidelines to inform EHR design and implementation. ${ }^{4}$ To develop these guidelines, it is essential to evaluate how EHRs are used in context.

This study quantitatively examined how physicians interacted with EHRs in primary care environments. The purpose of this study was to understand how physicians physically interacted with EHRs while providing care to the patients who reported high levels of satisfaction with the physician and the physician's EHR use. The study used quantified observational, where real encounters were videotaped and reduced to measureable units that could be quantified. Study findings will contribute to the design of EHRs and other health information technologies which can potentially compliment patient and physician capabilities and limitations. 


\section{Method}

\subsection{Data collection and sample}

100 patients and 10 physicians were recruited from five primary care clinics in 2011. All visits were recorded with high resolution video cameras. Informed consent was obtained from both patient and physician participants. The study protocol and activities were approved by university and clinic Institutional Review Boards and HIPAA (Health Insurance Portability and Accountability Act) regulations were fulfilled. Ten patients per physician were recruited; 56 male and 44 female comprised the patient group. 78 participants were White/Caucasian. Of the patients, 10 had some high school education, 27 were high school graduates, 24 had some college education, and 39 were college graduates. Patients were between 18 and 65 years old ( $M 45.2$ years old) and were patients of their primary care physicians for 1 to 38 years. Six male and four female physicians volunteered to participate in the study ( $M 47.6$ years old) and had been practicing family medicine for 5 to 37 years. The recruited physicians used computers in clinical consultations for 3 to 10 years.

\subsection{Data analysis}

\subsubsection{Variables}

Physician gaze at computer and typing were the variables of interest (see table 1). Duration of physician gaze at EHR, typing, and total communication time were calculated. The percentage of the physician's computer gaze and typing out of total communication time was also calculated with a mixed model. Survey questions evaluated patient assessment of their physician, and physicians' use of the EHR.

\subsubsection{Video coding}

Coding is the process of converting complex data into measurable units. ${ }^{13}$ A coding scheme was created for the variables of interest (see table 1). In the first stage of coding, each video was coded temporally for the entire visit length. Start and stop times for each code were annotated using software (Noldus Observer XT), which is designed for video coding, evaluation and analysis. The software calculated the start and stop times, duration, and simultaneous occurrence of codes (i.e. codes that occurred at the same time).

In the second stage of coding, codes related to interaction with EHR (typing) were classified evaluated for trends and potential classification. All videos were coded by five trained research assistants. Coders were trained to execute the coding procedures and reliability checks were conducted at regular intervals. Reliability scores for codes ranged from 0.62 to 0.88 , the range of standard and acceptable scores. ${ }^{14}$ The reliability time period was $\mathrm{X} \pm 1$ second, which is relatively conservative, in other word, if two coders coded the start of an event in the period of $\mathrm{X} \pm 1$, it was counted as an agreement.

Table 1. Coding scheme of the study

\begin{tabular}{|c|l|}
\hline Codes & Definition \\
\hline Subjects & \\
\hline Patient & The patient in the encounter. \\
\hline Physician & The primary care provider in the encounter. \\
\hline Behaviours & \\
\hline Gaze & Participant's head and/or body were in the direction of the target object. \\
\hline Typing & Participant used the keyboard to enter information. \\
\hline Object & \multicolumn{2}{|c|}{} \\
\hline Computer & The computer used in the medical visit. \\
\hline
\end{tabular}




\section{Results}

Physician gaze at computer varied from $24.9 \%$ to $49.6 \%$ between groups (see table 2 ). Typing time also varied ranging from $2.8 \%$ to $21.6 \%$ between groups. Percentage values for the occurrence of each code were estimated as duration of the behaviour.

Three distinct classifications of physician interactive behaviours emerged from the data; technology-centred, optimizing, and human-centred. Interactive behaviours were classified based on the percentage of typing in the visits; technology-centred $>15 \%$, optimizing $5 \%-15 \%$, and human-centred $<$ $5 \%$ (see table 2). To validate the classification, the relationship between typing time and classification was evaluated; significant differences in typing time were found between the technology-centred and human-centred groups $(p=0.00)$, technology-centred and optimizing groups $(\mathrm{p}=0.00)$, and optimizing and human-centred groups $(p=0.00)$. There was also a significant difference between physician time gazing at computer and classification group; technologycentred and human-centred $(\mathrm{p}=0.00)$, technologycentred and optimizing $(\mathrm{p}=0.00)$, and optimizing and human-centred $(\mathrm{p}=0.00)$. In addition, patient assessments of physicians EHR use were obtained with survey items, using a 5-point likert response scale, five indicating high levels of the construct. The table shows that all physicians received high ratings of patient trust, patient trust in their EHR use as well as, high patient satisfaction with their EHR use $(>4$ out of 5).

Table 2. Mean ratings for physician interaction and patient assessments

\begin{tabular}{|c|c|c|c|c|c|c|c|c|c|}
\hline \multirow[t]{2}{*}{$\begin{array}{c}\text { Interactive } \\
\text { style }\end{array}$} & \multirow[t]{2}{*}{ Visits } & \multirow{2}{*}{$\begin{array}{c}\text { Total } \\
\text { communicatio } \\
\boldsymbol{n} \\
\text { time }\end{array}$} & \multicolumn{2}{|c|}{$\begin{array}{c}\text { MD gaze at } \\
\text { computer }\end{array}$} & \multicolumn{2}{|c|}{ Typing } & \multirow{2}{*}{$\begin{array}{c}\begin{array}{c}\text { Patient } \\
\text { trust in } \\
\text { physician }\end{array} \\
\text { Mean } \\
\text { rating }\end{array}$} & \multirow{2}{*}{$\begin{array}{c}\text { Patient trust } \\
\text { in physicians' } \\
\text { EHR use* } \\
\text { Mean rating }\end{array}$} & \multirow{2}{*}{$\begin{array}{c}\text { Patient } \\
\text { satisfaction of } \\
\text { physicians' EHR } \\
\text { use * }\end{array}$} \\
\hline & & & $\begin{array}{l}\text { Time } \\
(\mathrm{sec})\end{array}$ & $\%$ & $\begin{array}{l}\text { Time } \\
(\mathrm{sec})\end{array}$ & $\%$ & & & \\
\hline $\begin{array}{c}\text { Technology } \\
\text { Centered }\end{array}$ & 20 & 1449.75 & 717.78 & $\begin{array}{c}49.6 \\
\%\end{array}$ & 269.27 & $\begin{array}{c}21.6 \\
\%\end{array}$ & 4.55 & 4.59 & 4.59 \\
\hline Optimizing & 40 & 1011.25 & 348.64 & $\begin{array}{c}34.8 \\
\%\end{array}$ & 84.29 & $8.5 \%$ & 4.73 & 4.69 & 4.67 \\
\hline $\begin{array}{l}\text { Human } \\
\text { Centered }\end{array}$ & 40 & 924.45 & 225.16 & $\begin{array}{c}24.9 \\
\%\end{array}$ & 29.04 & $2.8 \%$ & 4.66 & 4.43 & 4.62 \\
\hline
\end{tabular}

Note: $*$ out of 5 likert scale

\subsection{Detailed descriptions of each group}

Based on patterns identified across visits, interactions could be linked to individual physicians. For example, if the total number of a physician's visits were classified as technology-centred, that physician was classified by the interactive characteristics of that group. Physicians with humancentred styles tended to be older (51 to 69 years old), while physicians in other two groups were younger (37 to 44 years old).

Physicians in the technology-centred group $(n=2)$ typed the most out of the three groups; they spent $21.6 \%$ of the visit typing and gazed at the computer $49.6 \%$ of the time. They typed continuously throughout the visit $(269.27$ seconds), compared to physicians in the optimizing group who typed periodically in the visit (84.29 seconds). Descriptively, physicians in technology-centred group had a tendency to multitask; activities included gazing at the patient while typing, talking to patients while gazing at the monitor, verbal and nonverbal backchannels such as affirmative speech (e.g. "ok",
"I see", "mm hmm") and nodding. They also tended to type quickly using a touch typing style, which is typically used by individuals with expert typing abilities. $^{15}$

Physicians in the optimizing group $(\mathrm{n}=4)$ typed $8.5 \%$ of the visit and gazed at the computer $34.8 \%$ of the visit. Descriptively, participants in this group did not multitask as much as physicians in the technology-centred group. Physicians in the optimizing group tended to interact with EHR through brief, short typing sessions where they focused solely on the EHR. When they spoke to patients they tended to stop typing and gaze at patient. This interactive style may have also minimized the possibility of making typing mistakes. Physicians in this group also maintained positive nonverbal behaviours such as eye contact and a postural style that allowed the physician to face the patient most of the time.

Physicians in the human-centred group $(n=4)$ typed the least at $2.8 \%$ of the visit and gazed at the computer $24.9 \%$ of the visit. Descriptively, this group tended to have less developed typing skills and typed 
more slowly using a hunt and peck style characteristic of novice typers. ${ }^{15}$ These physicians also used aids to help manage data entry such as paper charts, nurse transcriptionists and voice dictation. Physicians in this group had higher amounts of positive verbal and nonverbal communication style with the patient than physicians in the technology-centred and optimizing groups.

\section{Discussion}

Physicians in this study received high ratings of patient trust ( $M 4.64$ out of 5 points) and patient satisfaction with their use of EHRs ( $M 4.62$ out of 5 points). Results show that these physicians had different styles of interacting with EHRs, which were called, technology-centred, human-centred and optimizing. Because each of these groups received high ratings of patient satisfaction, it may indicate that a variety of different human-technology interactive styles may be effective for physician interaction with HIT while providing patient care.

Physical interaction with EHR was primarily in the form of typing, though physicians also interacted with the technology through gaze. Qualitatively, typing was used to input information (i.e. data entry), while gaze was used to extract information (i.e. find information). Results show a relationship between the amount of time a physician interacted through typing and the amount of time they interacted through gaze. Physicians who typed for higher percentages of the visit also tended to gaze at the computer more, while physicians who typed for smaller percentages of the visit tended to gaze at the computer less.

Physicians in the technology-centred group tended to provide positive verbal and nonverbal communication while interacting with the EHR. They used verbal and non-verbal backchannels to illustrate that they were listening. It is possible that inputting information might also be an effective method of showing the patient that they are being heard. In contrast, physicians in the optimizing group switched their attention from the patient to the EHR throughout the visit; they also provided positive verbal and nonverbal communication during the times they focused on the patient. In contrast to the optimizing and technology-centred groups, physicians in the human-centred group spent the majority of their time focusing on the patient and relied on aids and post-visit time to input data.

It was expected that all physicians would have the same amount of gaze at screen to extract information from patients during the visits. The results show that technology-centred physicians spent the most time inputting and extracting information, which may illustrate an inability to interact with the technology at high levels and recall information about the patient. One advantage of this style of interaction is that it may be more efficient, in terms of time spent interacting with patient and inputting necessary data. This type of interaction may be problematic in situations where it may be necessary to provide care without technologies. ${ }^{16}$ Other disadvantages are that this group may not be able to communicate empathy appropriately, when necessary, using the methods they tend to use (multitasking with short verbal and nonverbal interactions). Other potential disadvantages may be that the cognitive demands associated with simultaneous data entry, data extraction and communication may lead to ineffective allocations between the three. This potential problem has been described in previous EHR interaction studies. ${ }^{9,11}$ For example, physicians in the technology-centred group relied on the EHR to extract information about the patient more than the other groups (i.e. they had twice as much gaze at computer when compared to the human-centred group). This over-reliance on technology to provide information may be because it was difficult for them to remember information about the patient, recall details or maintain active engagement in the conversation while typing.

Physicians in the optimizing group tended to switch their interactions with the technology with patient-centred interactions throughout the visit. Physicians that used this method also received high ratings of patient satisfaction. Some of the major risks with this interaction are that the physicians might over-rely or under-rely on the technology, falling into the technology-centred or human-centred interactive styles. This can also be considered a benefit as this can reduce potential burn-out, since physicians can control how much attention they give to the EHR and the patient respectively.

Finally, physicians in the human-centred group tended to under-rely on the technology. They had a tendency to focus their interactions in the visit on the patient completely, using a variety of aides to accomplish the necessary task of entering the data from the visit into the record. This interaction may have been the most costly in terms of time and cost. In order to keep the record up to date, physicians entered data after the visit and utilized aides such as transcriptionists. Some of the benefits of this style are that the patients received high levels of patientcentred care, in terms of eye contact and through listening. What is interesting is that this group of physicians also had lower levels of gaze at the computer, which may indicate that they did not need to extract information about the patients from the EHR as much as the physicians in the optimizing and technology-centred group. This could indicate that 
human-centred physicians knew their patients better, were better able to remember important information or that they were interested in information that could not be found in the EHR. One risk of this interactive style is that physicians might lose or forget important information before it is inputted into the record. Secondly, these physicians may be more prone to burnout, because they must be affectively engaged through the entire visit and they must rely heavily on their memory to communicate with the patient. Finally, they may not be fully benefitting for EHRs and other HITs that may enhance the quality of care they are able to provide.

These findings illustrate that different methods of interaction with technology can be effective in contributing to patient-centred outcomes. However, each method may have short and long term risks. Data regarding these potential risks was not collected in this study, but should be explored in future studies.

It is essential to identify effective strategies to integrate EHRs into clinical practice. ${ }^{9}$ Future EHR designs and training systems should consider flexible systems that accommodate the variety of interactive styles that physicians in this study used to provide patient-centred care. Future work in the area should evaluate new technologies that afford each of these interactive styles. This research will be increasingly important as EHR and HITs become more pervasive and include larger amounts of data, such as data from the home or consumer health technologies.

\section{Acknowledgements}

The University of Wisconsin- Madison Systems Engineering Initiative for Patient Safety (SEIPS) provided support on this project (http://cqpi.engr.wisc.edu). We thank undergraduate research assistants who assisted with data analysis and graduate research assistants who assisted with data collection.

\section{References}

[1] Fang H, Peifer K, Chen J, Rizzo J. Health information technology and physicians' perceptions of healthcare quality. The American journal of managed care. 2011;17(3):e66.
[2] Chaudhry B, Wang J, Wu S. Systematic review: impact of health information technology on quality, efficiency, and costs of medical care. Annals of internal medicine. 2006;144(10):742.

[3] Blumenthal D. Stimulating the adoption of health information technology. New England Journal of Medicine. 2009;360(15):1477-1479.

[4] Stead WW, Lin H. Computational technology for effective health care: immediate steps and strategic directions: Natl Academy Pr; 2009.

[5] Garrison GM, Bernard ME, Rasmussen NH. 21st-century health care: the effect of computer use by physicians on patient satisfaction at a family medicine clinic. Family Medicine-Kansas City-. 2002;34(5):362-368.

[6] Ong L, De Haes J, Hoos A, Lammes F. Doctor-patient communication: a review of the literature. Social Science \& Medicine. 1995;40(7):903-918.

[7] Roter D, Frankel R, Hall J, Sluyter D. The expression of emotion through nonverbal behavior in medical visits. Journal of General Internal Medicine. 2006;21(S1):S28-S34.

[8] Montague E, Xu J, Chen P-y, Asan O, Barrett B, Chewning B. Modeling eye gaze patterns in clinician-patient interaction with lag sequential analysis. Human Factors. in press.

[9] Shachak A, Reis S. The impact of electronic medical records on patient-doctor communication during consultation: a narrative literature review. Journal of Evaluation in Clinical Practice. 2009;15(4):641-649.

[10] Lelievre S, Schultz K. Does computer use in patient-physician encounters influence patient satisfaction? Canadian Family Physician. 2010;56(1):e6.

[11] Margalit RS, Roter D, Dunevant MA, Larson S, Reis S. Electronic medical record use and physician-patient communication: an observational study of Israeli primary care encounters. Patient education and counseling. 2006;61(1):134141.

[12] Holden RJ. Social and personal normative influences on healthcare professionals to use information technology: towards a more robust social ergonomics. Theoretical Issues in Ergonomics Science. 2011;99999(1):1-24.

[13] Miles MB, Hubberman AM. Data management and analysis methods. In: Denzin NK, Lincoln YS, eds. Qualitative data analysis: An expanded sourcebook. 2nd ed. CA: Thousand Oaks; 1994:428-444.

[14] Bakeman R. Behavioral observation and coding. Handbook of research methods in social and personality psychology. 2000:138-159.

[15] Goldberg D, Richardson C. Touch-typing with a stylus. Paper presented at: INTERACT '93 and CHI '93 conference on Human factors in computing systems, 1993; New York, NY.

[16] Singh R, Lichter MI, Danzo A, Taylor J, Rosenthal T. The Adoption and Use of Health Information Technology in Rural Areas: Results of a National Survey. The Journal of Rural Health. 2011:1-12. 